\title{
An updating method for structural dynamics models with uncertainties
}

\author{
B. Faverjon, P. Ladevèze ${ }^{* *}$ and F. Louf* \\ LMT-Cachan (ENS Cachan/CNRS UMR8535/Paris 6 University), 61, avenue du Président Wilson, F-94235 \\ Cachan Cedex, France
}

Received 2007

Revised 2007

\begin{abstract}
One challenge in the numerical simulation of industrial structures is model validation based on experimental data. Among the indirect or parametric methods available, one is based on the "mechanical" concept of constitutive relation error estimator introduced in order to quantify the quality of finite element analyses. In the case of uncertain measurements obtained from a family of quasi-identical structures, parameters need to be modeled randomly. In this paper, we consider the case of a damped structure modeled with stochastic variables. Polynomial chaos expansion and reduced bases are used to solve the stochastic problems involved in the calculation of the error.
\end{abstract}

Keywords: Damping, validation, dissipation, constitutive relation error, stochastic model, polynomial chaos

\section{Introduction}

Industrial problems are often solved by numerical simulations involving very complex models. In addition, tests are carried out in order to ensure that the complexity of the structures is properly accounted for. Usually, only a small set of measurements is performed in order to validate the numerical model. Very often, the test data and numerical predictions are poorly correlated, which can make it difficult to model some parts of structures whose behavior is not known very well. When the correlation between numerical simulations and experimental data is unsatisfactory, model updating methods are used. The purpose of these methods is to minimize the discrepancy between the test data and the numerical model by modifying the latter. A state-of-the-art review of these methods can be found in [1].

Unfortunately, in the case of what are known as 'direct methods' [2,3], the corrections to the model's mass and stiffness matrices do not take into account the physical meaning of the modifications. Conversely, indirect or parametric methods lead to updated mass and stiffness matrices obtained through changes in the physical parameters of the model. The associated cost functions can be classified into three categories: input residuals [4,5], output residuals [6,7] and the specific residual called "Constitutive Relation Error (CRE)". The last residual, which is used in this paper, provides a measure of the quality of the updated model, which is essential for model validation. This approach, which uses the concept of Drucker error, provides an effective means of updating mass and stiffness matrices $[9,10]$. The first developments of this method [8] showed the effectiveness of such a model in structural dynamics, then in forced vibrations with updated models obtained from eigenmodes.

An extension of this approach to the stochastic case was given in [11,12] and applied particularly to the resolution of simple structural dynamics problems. The case of uncertain measurements obtained from a family of quasiidentical structures requires the parameters to be modeled randomly. Here, since the problem consists in deriving an

\footnotetext{
*Corresponding author. E-mail: louf@1mt.ens-cachan.fr.

**EADS Foundation Chair "Advanced Computational Structural Mechanics".
} 


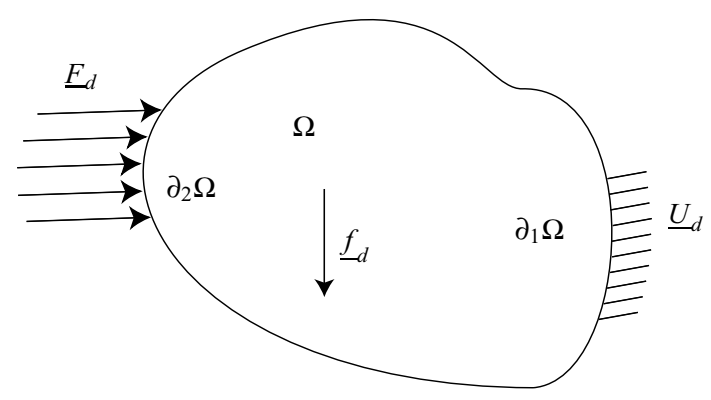

Fig. 1. The domain being studied and the applied loads.

error measure which is zero if the model is "exact", i.e. if the results obtained from the model match the experimental data, the main difficulty resides in the validation of the stochastic model. Since the statistical description of the experimental data is often very limited, we proposed to reconstruct these experimental data using the statistical mean value and the model itself $[11,12]$. This reconstruction was based on a qualitative analysis of the error in the response in terms of two sources of errors, which are the fluctuations of the parameters and the possible variations due to the approximate nature of the stochastic model itself.

In [14], we considered the case of complex engineering structures involving large numbers of stochastic variables. Polynomial chaos expansion [15] and reduced bases were used to solve the stochastic problems involved in the calculation of the error.

In this paper, a variant of the method, based on the dissipation error introduced in [16,17], is presented. The main difference between this error and the Drucker error lies in the choice of the reference. The dissipation error assumes that all the equations are verified except for the evolution laws. Thus, this new approach focuses on the structure's damping.

Section 2 of the paper presents the theoretical aspects of model validation based on the extension of both the dissipation error and the error in the measurements to the stochastic case. Section 3 deals with the discretization of the stochastic error. The problem of the minimization of the stochastic error is described and solved using polynomial chaos expansion [15]. Finally, Section 4 presents an application of the stochastic model updating method: The Drucker error is used to update the mass and stiffness; then, the dissipation error is used to update the stochastic damping model parameters.

\section{Presentation of the updating method}

\subsection{The problem to be solved}

Let us consider the vibrations of a structure $\Omega$ over a time interval $t \in[0, T]$ (see Fig. 1). A force distribution $\underline{F} d$ is applied to a part $\partial_{2} \Omega$ of the boundary of the structure and a displacement $\underline{U}_{d}$ is prescribed over the complementary part $\partial_{1} \Omega$, with $\partial \Omega=\partial_{1} \Omega \cup \partial_{2} \Omega$. The forces $\underline{f}_{d}$ are body forces in Domain $\Omega$. The model is probabilistic. In the Karhunen-Love method, the model's parameters and the prescribed quantities are stochastic variables [15,18]. Let us consider two random variable vectors $\underline{m}(\theta)$ and $\underline{d}(\theta)$, whose probability density functions are $d P(\underline{m})$ (resp. $d Q(\underline{d}))$ and whose corresponding spaces are $H_{m}$ (resp. $\left.H_{d}\right)$. The physical parameters of the model are functions of $\underline{m}(\theta)$, while $\underline{F}_{d}, \underline{U}_{d}$ and $\underline{f}_{d}$ depend on $\underline{d}(\theta)$; the argument $\theta$ denotes the random character of the corresponding quantity. $M \in \Omega$ being the position vector, the problem to be solved is to find the solution $s(\underline{M}, t, \underline{m}(\theta), \underline{d}(\theta))$ of a parameterized problem whose parameters are $\underline{m}$ and $\underline{d}$. Since this paper deals with free and forced vibration problems, the equations will be written in the frequency domain.

Thus, the reference problem consists in finding $(\underline{M}, t, \underline{m}(\theta), \underline{d}(\theta))=(\underline{U}(\underline{M}, t, \theta), \sigma(\underline{M}, t, \theta), \Gamma(\underline{M}, t, \theta))$ (the displacement, the stress and the acceleration respectively, $M \in \Omega$ being the position vector) which verify the kinematic constraints, the equilibrium equations and the constitutive equations. The concept of error in constitutive relation is based on the separation of the reliable equations or quantities from the less reliable equations or quantities. 
What belongs to one category or the other depends on the error being used. In this paper, we will work with the "dissipation error", which was introduced in previous works (see for example $[16,17]$ ) and was shown to be more effective than another error, the Drucker error, in the context of the updating of damping. The last section of the paper will give elements of comparison between the two types of errors. For stochastic problems using the Drucker error, please refer to $[11,12,14]$.

For the sake of simplicity, the dependencies on stochastic variables will be omitted throughout the rest of this paper, except when necessary.

\subsection{The dissipation stochastic constitutive relation error}

For the dissipation error, the reliable equations consist in the kinematic constraints, the equilibrium equations and the state laws; the less reliable equations are those which describe dissipation. A solution $s(\underline{m}, \underline{d})$ is said to be admissible if $\forall \underline{d} \in H_{d}$ it verifies the equations which are considered reliable. In this case, the constitutive relation error, which represents the modeling dissipation error $\zeta_{\omega}^{2}$ in a stochastic sense, is given by

$$
\zeta_{\omega}^{2}(\underline{U}, \underline{V})=\int_{H_{m} \times H_{d}} \frac{T \omega^{2}}{4} \int_{\Omega} \operatorname{tr}\left[\mathbf{B}\left(\epsilon(\underline{V})-\epsilon(\underline{U})^{\star}(\epsilon(\underline{V})-\epsilon(\underline{U}))\right] d \Omega d P(\underline{m}) d Q(\underline{d}),\right.
$$

in which $X^{\star}$ denotes the complex conjugate of Quantity $X$. The solution $s$ can also be written as $s^{\prime}=(\underline{U}, \underline{V})$, where Fields $\underline{U}$ and $\underline{V}$ correspond to dependencies of the strain and stress tensors $\epsilon=\epsilon(\underline{U})$ and $\sigma=i \omega \mathbf{B} \epsilon(\underline{V})$ respectively, $\omega$ being the angular frequency and $\mathbf{B}$ the damping operator.

\subsection{Error in the measurements and modified dissipation error}

In the context of model updating, additional data coming from measurements on several real structures are used. Again, these quantities are subdivided into a set of reliable equations (the measured angular frequency $\omega$, the positions and directions of the excitations and sensors) and a set of less reliable equations (the amplitudes of the forces $\underline{\tilde{F}}_{d}(\tilde{d}(\theta))$ and displacements $\underline{\tilde{U}}_{d}(\tilde{d}(\theta))$ at the excitation and sensor points). In the rest of the paper, measured quantities will be designated by the notation $\tilde{\boldsymbol{~}}$.

The influence of these less reliable quantities is taken into account by a specific measurement error term which, at Frequency $\omega$, is given by:

$$
\eta_{\omega}^{2}=\int_{H_{m} \times H_{d}}\left(\frac{\left\|\left.\underline{U}\right|_{\partial_{1} \Omega}-\underline{\tilde{U}}_{d}\right\|^{2}}{\left\|\underline{\tilde{U}}_{d}\right\|^{2}}+\frac{\left\|\left.\underline{F}\right|_{\partial_{2} \Omega}-\underline{\tilde{F}}_{d}\right\|^{2}}{\left\|\underline{\tilde{F}}_{d}\right\|^{2}}\right) d P(\underline{m}) d \tilde{Q}(\underline{\tilde{d}})
$$

where $d \tilde{Q}(\underline{\tilde{d}})$ is the probability density function associated with Space $H_{d}$. Then, the problem consists in finding admissible fields $(\underline{U}, \underline{V})$ which verify the less reliable equations as closely as possible in order to minimize the modified dissipation error defined by:

$$
e_{\omega}^{2}=\frac{\zeta_{\omega}^{2}}{D_{\omega}^{2}}+\frac{r}{1-r} \eta_{\omega}^{2}
$$

where $r$ is a weighting coefficient representing our degree of trust in the experimental data. Previous works [12] showed that Coefficient $r$ can be chosen equal to 0.5. The denominator $D_{\omega}^{2}$ and the norms being used ensure that the two error terms have equivalent weights. Over the whole frequency range $\left[\omega_{\min }, \omega_{\max }\right]$, the modified dissipation error is calculated by introducing a weighting factor $z(\omega)$ such that $\int_{\omega_{\min }}^{\omega_{\max }} z(\omega) d \omega=1$, with $z(\omega) \geqslant 0$ (e.g. $\left.z(\omega)=1 /\left(\omega_{\max }-\omega_{\min }\right)\right)$, and is expressed as

$$
e_{T}^{2}=\zeta_{T}^{2}+\eta_{T}^{2} \quad, \quad \text { with } \quad \zeta_{T}^{2}=\int_{\omega_{\min }}^{\omega_{\max }} \frac{\zeta_{\omega}^{2}}{D_{\omega}^{2}} z(\omega) d \omega \quad \text { and } \quad \eta_{T}^{2}=\int_{\omega_{\min }}^{\omega_{\max }} \eta_{\omega}^{2} z(\omega) d \omega .
$$

Let us note that previous works [19] showed that the following expression is properly balanced:

$$
D_{\omega}^{2}=\int_{H_{m} \times H_{d}} \frac{T \omega^{2}}{2} \int_{\Omega} \operatorname{tr}\left[\mathbf{B} \epsilon(\underline{U})^{\star} \epsilon(\underline{U})\right] d \Omega d P(\underline{m}) d Q(\underline{d}) .
$$




\subsection{Discretized form of the modified error and reconstruction of the experimental data}

In order to proceed further, let us now consider a discretized form of the modified error. A detailed development of what follows can be found in [11,12]. The problem to be solved is: find $\underline{S}$ (the discrete form of the boundary vector $\left(\left.\underline{U}\right|_{\partial_{1} \Omega},\left.\underline{F}\right|_{\partial_{2} \Omega}\right)$ ) which minimizes:

$$
\begin{aligned}
\underline{S} & \rightarrow e_{\omega}^{2}(\underline{S}) \\
\mathbb{R}^{n} & \rightarrow \mathbb{R},
\end{aligned}
$$

with

$$
e_{\omega}^{2}(\underline{S})=\int_{H_{m} \times H_{d}}\left(\underline{S}^{\star T} \mathbb{H} \underline{S}+\frac{r}{1-r}(\underline{S}-\underline{\tilde{S}})^{\star T} \mathbb{Z}(\underline{S}-\underline{\tilde{S}})\right) d P(\underline{m}) d Q(\underline{d}),
$$

all the admissible constraints being taken into account through Operators $\mathbb{H}$ and $\mathbb{Z}$. Superscript $T$ denotes the transpose operator. Besides, one has

$$
\left(\mathbb{H}+\frac{r}{1-r} \mathbb{Z}\right) \underline{S}=\frac{r}{1-r} \mathbb{Z} \underline{\tilde{S}},
$$

where Operator $\mathbb{H}+\frac{r}{1-r} \mathbb{Z}$ is regular.

Although the experimental data $\underline{\tilde{S}}$ are random quantities, we assume that these are not affected by measurement errors. The "exact" solution of the stochastic model is given by:

$$
\mathbb{H} \underline{S}=0 \text {. }
$$

The difficulty in deriving error estimators for validation purposes resides in the fact that for an "exact" stochastic model the error should be zero. This means that for any excitation the probability density function of $\underline{S}$ calculated by the model should be equal to that derived from experimental data. The objective is to describe the experimental values (which, in practice, are very sparse) and compare them with the values given by the model. It should be noted that there are two sources of perturbations, one associated with the model itself and the other related to the fluctuations inherent in the stochastic nature of the model. For a nearly identical model, neglecting the second-order terms, the solution calculated by the model can be written as [12]:

$$
\underline{S}(\underline{m}(\theta))=\underline{\bar{S}}+\mu \Delta \underline{S}+\mu^{\prime}\left[\begin{array}{c}
(\mathbb{Q}-\overline{\mathbb{Q}}) \underline{S}_{I} \\
0
\end{array}\right]
$$

where $\overline{\boldsymbol{}}=\int_{H_{m}} \bullet d P(\underline{m})$ and $\mu$ and $\mu^{\prime}$ are indicators, $\Delta$ denotes the difference between two quantities, $\mathbb{Q}$ is an operator which can be easily deduced from Eq. (9), and $\underline{S}_{I}$ is the excitation (assumed to be perfectly known) which gives the model a unique response such as $\underline{S}_{R}=\mathbb{Q}(\underline{m}(\theta)) \underline{S}_{I}$. The solution of an "exact" model is calculated with $\mu=0$. This means that on first-order approximation the errors in the model itself affect the mean value and nothing else. Very little statistical information is available experimentally. When the excitation is deterministic and the measurements are noise-free, it seems reasonable to use the mean value alone. Consequently, the reconstructed experimental data is given by:

$$
\underline{\tilde{S}}_{r e c}=\langle\langle\underline{\tilde{S}}\rangle\rangle+\left[\begin{array}{c}
(\mathbb{Q}(\underline{m}(\theta))-\overline{\mathbb{Q}}) \underline{\tilde{S}}_{I} \\
0
\end{array}\right], \quad \text { where }\langle\langle\bullet\rangle\rangle=\int_{H_{d}} \bullet d \tilde{Q}(\underline{\tilde{d}}) .
$$

\subsection{The updating process}

For each frequency $\omega$, the problem to be solved is expressed by Eq. (6). Having obtained the error $\zeta_{T}^{2}$ whose value represents the relative quality (in \%) of the numerical model with respect to measurements over a frequency range, one has to decide whether model updating is necessary. If it is, one must first perform the localization step, which consists in determining the "most erroneous" substructures $E$, whose model errors are $\zeta_{E T}^{2}$ such that

$$
\zeta_{E T}^{2} \geqslant \tilde{\delta} \max _{E \in E} \zeta_{E T}^{2}
$$


where for example $\tilde{\delta}=0.8$. Only the structural parameters belonging to these substructures are candidates for correction. Let us arrange these parameters into a vector $\underline{k}$. The next step is the correction step, in which the problem is solved by minimizing Functional $J(\underline{k})=e_{T}^{2}$. This is a nonlinear problem with respect to Parameters $\underline{k}$ which is solved through a gradient-based minimization algorithm. For each variation of Parameters $\underline{k}$, the mass, stiffness and damping matrices are reassembled. Once the correction has been made, the model error $\zeta_{T}^{2}$ is recalculated. If the new value is smaller than a given threshold, the updating process is terminated; otherwise, a new iteration consisting of a localization step and a correction step is performed.

\section{Discretization of the stochastic problem}

\subsection{The discrete problem}

In the remainder of this paper, we will consider the particular case in which the forces and displacements at the excitation points and sensor points are deterministic while the material parameters are stochastic. Thus, the only probabilistic space used will be $H_{m}$. For the sake of simplicity, dependence on $\underline{m}(\theta)$ will be denoted simply $\theta$. Moreover, in the case of a single excitation, the measured displacements will be normalized by the amplitude of the force vector, so only the amplitudes of the displacements appear in the expression of the error measure $\eta_{\omega}^{2}$ and Eq. (2) depends on the displacements alone. The discrete form of $e_{\omega}^{2}$ with respect to the vectors (denoted $\{U\}$ and $\{V\}$ ) of the nodal values of the stochastic displacement fields $\underline{U}$ and $\underline{V}$ is given by

$$
\begin{aligned}
e_{\omega}^{2}(\{U(\theta)\},\{V(\theta)\})= & \frac{T \omega^{2}}{4}\{U(\theta)-V(\theta)\}^{T \star}[\mathbf{B}(\theta)]\{U(\theta)-V(\theta)\} \\
& +\frac{r}{1-r}\{\Pi U(\theta)-\widetilde{U}\}^{T \star}[\mathbf{G}(\theta)]\{\Pi U(\theta)-\widetilde{U}\}
\end{aligned}
$$

where the notation $\{U(\theta)-V(\theta)\}$ is equivalent to $\{U(\theta)\}-\{V(\theta)\}$. $\Pi$ is a projection operator which, when applied to vector $\{X\}$, gives the value of this vector at the sensors. Besides, Matrix $[\mathbf{B}]$ is the damping matrix of the system and Matrix $[\mathbf{G}]$ quantifies the error in the measurements and can be taken as

$$
[\mathbf{G}]=\frac{T \omega^{2}}{4} \overline{[\mathbf{b}(\theta)]}
$$

where $\overline{[\mathbf{b}(\theta)]}$ is the system's mean reduced damping matrix at the measurement points. In addition, the solution $(\{U(\theta)\},\{V(\theta)\})$ must be admissible, i.e. it must verify

$$
\left([\mathbf{K}(\theta)]-\omega^{2}[\mathbf{M}(\theta)]\right)\{U(\theta)\}+i \omega[\mathbf{B}(\theta)]\{V(\theta)\}=\{F\},
$$

where $\{F\}$ is the excitation force vector, and $[\mathbf{K}]$ and $[\mathbf{M}]$ are the stiffness and mass matrices respectively. Finally, the minimization of Error $e_{\omega}^{2}$ under the admissibility constraints is obtained by introducing Lagrange multipliers, which leads to the resolution of the system of linear equations

$$
[\mathbf{A}(\theta)]\{X(\theta)\}=\{B\},
$$

with $[\mathbf{A}(\theta)],\{X(\theta)\}$ and $\{B\}$ given by

$$
\begin{aligned}
{[\mathbf{A}(\theta)] } & =\left[\begin{array}{cc}
\frac{T \omega^{2}}{2}[\mathbf{B}(\theta)]-i \frac{T \omega}{2}\left([\mathbf{K}(\theta)]-\omega^{2}[\mathbf{M}(\theta)]\right) & 2 \frac{r}{1-r} \Pi^{T}[\mathbf{G}] \Pi \\
-i \omega[\mathbf{B}(\theta)] & {[\mathbf{K}(\theta)]+i \omega[\mathbf{B}(\theta)]-\omega^{2}[\mathbf{M}(\theta)]}
\end{array}\right], \\
\{X(\theta)\} & =\left[\begin{array}{c}
\{U(\theta)-V(\theta)\} \\
\{U(\theta)\}
\end{array}\right] \quad \text { and } \quad\{B(\theta)\}=\left[\begin{array}{c}
2 \frac{r}{1-r} \Pi^{T}[\mathbf{G}]\{\tilde{U}(\theta)\} \\
\{F\}
\end{array}\right] .
\end{aligned}
$$




\subsection{Reduction of the model}

During the updating process, Problem (16) must be solved once for each frequency at each localization step, and many times at each correction step. Thus, for industrial structures with large numbers of degrees of freedom, the updating process can be very costly. An alternative is to use a reduced basis. Previous works $[13,14]$ showed that a significant cost reduction can be achieved using a reduced basis constructed from the constitutive relation error based on the Drucker error and adapted to large-scale stiffness updating problems in the aerospace field. In this paper, we present the reduced basis associated with the dissipation error. The pair of vectors $(\{U(\theta)\},\{U(\theta)-V(\theta)\})$ solution of Eq. (16) is also the solution of two forced vibrations problems:

$$
\begin{aligned}
& \left([\mathbf{K}(\theta)]-\omega^{2}[\mathbf{M}(\theta)]\right)\{U(\theta)-V(\theta)\}=\left\{F_{1}\right\}+\left\{F_{2}\right\}, \\
& \left([\mathbf{K}(\theta)]-\omega^{2}[\mathbf{M}(\theta)]\right)\{U(\theta)\}=\{F\}+\left\{F_{3}\right\},
\end{aligned}
$$

where

$$
\begin{aligned}
& \left\{F_{1}\right\}=-\frac{4 i}{T \omega} \frac{r}{1-r} \Pi^{T}[\mathbf{G}](\Pi\{U(\theta)\}-\{\widetilde{U}(\theta)\}), \\
& \left\{F_{2}\right\}=-i \omega[\mathbf{B}(\theta)]\{U(\theta)-V(\theta)\} \\
& \left\{F_{3}\right\}=-i \omega[\mathbf{B}(\theta)]\{V(\theta)\} .
\end{aligned}
$$

The random mass, stiffness and damping matrices $[\mathbf{M}(\theta)],[\mathbf{K}(\theta)]$ and $[\mathbf{B}(\theta)]$ are constructed using [15]:

$$
[\mathbf{X}(\theta)]=\sum_{i=1}^{N_{g}} \overline{[\mathbf{X}(\theta)]_{i}}\left(1+\delta_{i}^{X} \xi_{i}^{X}(\theta)\right),
$$

in which $N_{g}$ represents the number of substructures and $[\mathbf{X}(\theta)]$ designates $[\mathbf{M}(\theta)],[\mathbf{K}(\theta)]$ or $[\mathbf{B}(\theta)]$. Superscript $X=$ $M, K$, or $B$ is associated with $[\mathbf{X}(\theta)]$. Besides, $\xi_{i}^{X}(\theta)$ are the components of Vector $\underline{\xi}(\theta)$ of $L=N_{g} \times m$ Gaussian random variables $\left\{\left\{\xi_{i}^{M}(\theta)\right\}_{i=1}^{N_{g}},\left\{\xi_{i}^{K}(\theta)\right\}_{i=1}^{N_{g}},\left\{\xi_{i}^{B}(\theta)\right\}_{i=1}^{N_{g}}\right\}$ (in this case, there are $m=3$ material parameters). In addition, $\delta_{i}^{X}$ is the coefficient of variation related to the Gaussian variable $\xi_{i}^{X}(\theta)$, and $\overline{[\mathbf{X}(\theta)]}$ is the mathematical expectation of $[\mathbf{X}(\theta)]$. Then, Eqs (18) and (19) can also be written as follows:

$$
\begin{aligned}
& \left(\overline{[\mathbf{K}(\theta)]}-\omega^{2} \overline{[\mathbf{M}(\theta)]}\right)\{U(\theta)-V(\theta)\}=\{F\}_{I}, \\
& \left(\overline{[\mathbf{K}(\theta)]}-\omega^{2} \overline{[\mathbf{M}(\theta)]}\right)\{U(\theta)\}=\{F\}_{I I} .
\end{aligned}
$$

Thus, the reduced basis being used, denoted $[\mathbf{T}]$, consists in a truncated real modal basis constructed by taking the first $N$ real deterministic eigenmodes $\left\{\Phi_{i}\right\}, i=1$ to $N$, given by

$$
\left(\overline{[\mathbf{K}(\theta)]}-\omega_{i}^{2} \overline{[\mathbf{M}(\theta)]}\right)\left\{\Phi_{i}\right\}=\{0\},
$$

and the first term of a series of Krylov vectors associated with the excitations $\{F\}_{I},\{F\}_{I I}$, corresponding to the static response of the structure to these excitations. The excitation $\left\{F_{1}\right\}$ can be represented by unit forces $\left\{F_{s}\right\}_{k}$, $k=1$ to $N_{s}$ at each of the $N_{s}$ sensors. In $\left\{F_{2}\right\}$ and $\left\{F_{3}\right\}$, the unknown terms $\{V(\theta)\}$ and $\{U(\theta)-V(\theta)\}$ can be approximated by

$$
\begin{aligned}
& \{U(\theta)-V(\theta)\}=\sum_{i=1}^{N} a_{i}\left\{\Phi_{i}\right\}, \\
& \{V(\theta)\}=\sum_{i=1}^{N} b_{i}\left\{\Phi_{i}\right\},
\end{aligned}
$$

where $a_{i}$ and $b_{i}$ are coefficients. These terms are new static modes in the reduced basis related to the excitations $\left(i=1\right.$ to $N$ and $k=1$ to $N_{g}$ ) 
$\left.\left\{\overline{[\mathbf{B}]}\left\{\Phi_{i}\right\}, \overline{[\mathbf{B}}\right]_{k}\left\{\Phi_{i}\right\}\right\}$.

Since the matrices $[\mathbf{K}]$, $[\mathbf{B}]$ and $[\mathbf{M}]$ may change during the updating process, we have to add to the reduced basis the static response of the structure to the excitations associated with the variation of the mean material parameters $\Delta[\overline{\mathbf{M}}], \Delta[\overline{\mathbf{K}}]$ and $\Delta[\overline{\mathbf{B}}]$. The final choice of a reduced basis $[\mathbf{T}]$ contains the first $N$ real deterministic eigenmodes $\left\{\Phi_{i}\right\}$ and the deterministic static modes associated with the excitations

$$
\begin{aligned}
& \left.\left[\{F\},\left\{F_{s}\right\}_{k}, \overline{[\mathbf{B}]}\left\{\Phi_{i}\right\}, \overline{[\mathbf{K}}_{k}\left\{\Phi_{i}\right\}, \overline{\mathbf{M}}_{k}\left\{\Phi_{i}\right\}, \overline{[\mathbf{B}}\right]_{k}\left\{\Phi_{i}\right\}, \Delta[\overline{\mathbf{M}}]\left\{\Phi_{i}\right\}, \Delta[\overline{\mathbf{K}}]\left\{\Phi_{i}\right\}, \Delta[\overline{\mathbf{B}}]\left\{\Phi_{i}\right\}\right], \\
& \quad i=1 \text { to } N .
\end{aligned}
$$

Some of these vectors are very close to being collinear. Therefore, it is necessary to orthonormalize the reduced basis $[\mathbf{T}]$. This can be achieved using a Lanczos-type algorithm and may lead to the elimination of some vectors. One should note that the expression of the reduced basis $[\mathbf{T}]$ is similar to those constructed with the constitutive relation error (see [13,14]). Finally, the reduced quantities are identified by the subscript $r$ and are expressed as

$$
\begin{aligned}
& \{X(\theta)\}=[\mathbf{T}]\left\{X_{r}(\theta)\right\}, \quad \forall\{X(\theta)\} \text { equal to }\{U(\theta)\},\{V(\theta)\}, \\
& {\left[\mathbf{K}_{r}(\theta)\right]=[\mathbf{T}]^{T}[\mathbf{K}(\theta)][\mathbf{T}], \quad\left[\mathbf{M}_{r}(\theta)\right]=[\mathbf{T}]^{T}[\mathbf{M}(\theta)][\mathbf{T}],} \\
& {\left[\mathbf{B}_{r}(\theta)\right]=[\mathbf{T}]^{T}[\mathbf{B}(\theta)][\mathbf{T}], \quad\left\{F_{r}\right\}=[\mathbf{T}]^{T}\{F\}, \quad \Pi_{r}=[\mathbf{T}] \Pi,}
\end{aligned}
$$

The new reduced linear system, whose size is twice that of the reduced basis, is given by

$$
\left[\mathbf{A}_{r}\right]\left\{X_{r}\right\}=\left\{B_{r}\right\}
$$

where (omitting the dependence in $\theta$ ) $\left[\mathbf{A}_{r}\right],\left\{X_{r}\right\}$ and $\left[\mathbf{B}_{r}\right]$ are expressed as

$$
\left[\mathbf{A}_{r}\right]=\left[\begin{array}{cc}
\frac{T \omega^{2}}{2}\left[\mathbf{B}_{r}\right]-i \frac{T \omega}{2}\left(\left[\mathbf{K}_{r}\right]-\omega^{2}\left[\mathbf{M}_{r}\right]\right) & 2 \frac{r}{1-r} \Pi_{r}^{T}[\mathbf{G}] \Pi_{r} \\
-i \omega\left[\mathbf{B}_{r}\right] & {\left[\mathbf{K}_{r}\right]+i \omega\left[\mathbf{B}_{r}\right]-\omega^{2}\left[\mathbf{M}_{r}\right]}
\end{array}\right],
$$

with

$$
\left\{X_{r}\right\}=\left[\begin{array}{c}
\left\{U_{r}-V_{r}\right\} \\
\left\{U_{r}\right\}
\end{array}\right] \quad \text { and } \quad\left\{B_{r}\right\}=\left[\begin{array}{c}
2 \frac{r}{1-r} \Pi_{r}^{T}[\mathbf{G}]\{\widetilde{U}\} \\
\left\{F_{r}\right\}
\end{array}\right] .
$$

\subsection{The polynomial chaos expansion}

The random vectors $\{U(\theta)\}$ and $\{V(\theta)\}$ are expanded in terms of polynomial chaoses such as [15]

$$
\{X(\theta)\}=\sum_{j=0}^{\infty}\{X\}_{j} \Psi_{j}(\underline{\xi}(\theta)) .
$$

By truncating the expansion after the $P^{\text {th }}$ term, Eq. (36) yields

$$
\{X(\theta)\}=\sum_{j=0}^{P}\{X\}_{j} \Psi_{j}(\underline{\xi}(\theta)),
$$

where $\{X\}_{i}$ is the deterministic nodal solution $\{U\}_{i}$ or $\{V\}_{i}, i=0$ to $P . P$ is the total number of polynomial chaoses used in the expansion (excluding the $0^{t h}$-order term) and can be determined by $P=1+\sum_{s=1}^{p} \frac{1}{s !} \prod_{r=0}^{s-1}(L+r), p$ being the order of the homogeneous chaos being used [15]. $\left\{\Psi_{j}(\theta)\right\}_{j=0 \text { to } P}$ refers to a rearrangement of the $p^{t h}-$ order finite-dimension orthogonal polynomials with respect to the Gaussian function, which constitute a complete basis of the space of the second-order random variables. The substitution of Eq. (37) into the linear system Eq. (17) and its projection onto the subspace spanned by the polynomial chaos subset leads to a new system written as

$$
\left[\begin{array}{ccccc}
{\left[\mathbf{A}_{r,(00)}\right]\left[\mathbf{A}_{r,(01)}\right]} & \ldots & \ldots & {\left[\mathbf{A}_{r,(0 P)}\right]} \\
\cdot & \cdot & \ldots & \ldots & \cdot \\
\cdot & \cdot & {\left[\mathbf{A}_{r,(i j)}\right]} & \ldots & \cdot \\
\cdot & \cdot & \ldots & \ldots & \cdot \\
{\left[\mathbf{A}_{r,(P 0)}\right]\left[\mathbf{A}_{r,(P 1)}\right]} & \ldots & \ldots & {\left[\mathbf{A}_{r,(P P)}\right]}
\end{array}\right]\left[\begin{array}{c}
\left\{X_{0}\right\} \\
\cdot \\
\left\{X_{j}\right\} \\
\cdot \\
\left\{X_{P}\right\}
\end{array}\right]=\left[\begin{array}{c}
\left\{B_{r}\right\} \\
\cdot \\
\{0\} \\
\cdot \\
\{0\}
\end{array}\right],
$$




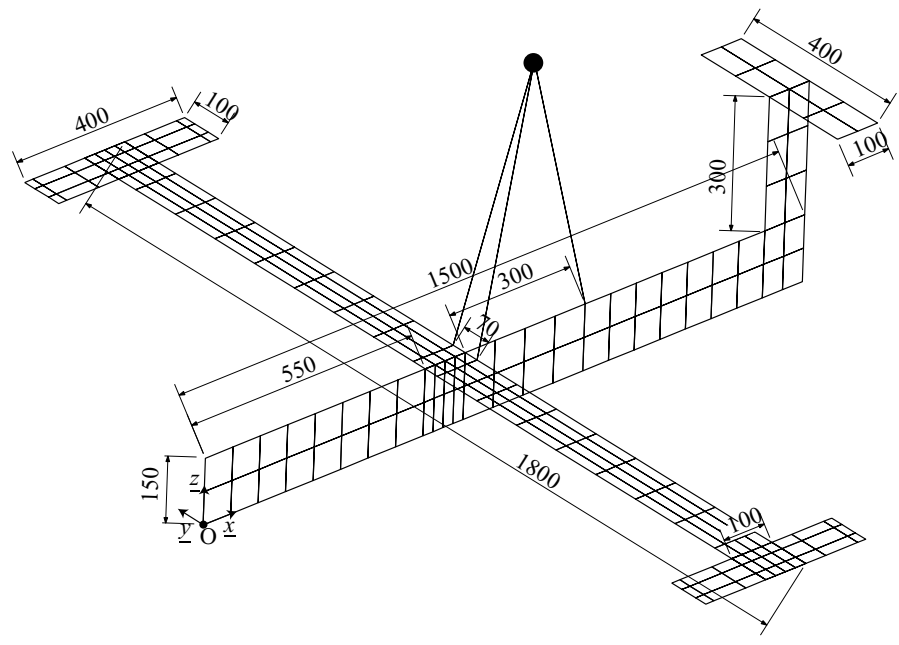

Fig. 2. Geometry of the test structure (dimensions in $\mathrm{mm}$ ).

where $\left[\mathbf{A}_{r,(i j)}\right]$ denotes the block matrix associated with the chaos indexes $i$ and $j(i, j=0$ to $P)$ and is given by

$$
\left[\mathbf{A}_{r,(i j)}\right](\omega)=\left\langle\Psi_{i}^{2}\right\rangle \delta_{i j} \overline{\left[\mathbf{A}_{r}\right]}(\omega)+\sum_{k=1}^{N_{g}} c_{i j k^{K}} \delta_{k}^{K}{\overline{\left[\mathbf{A}_{r}\right.}}_{k}^{K}+c_{i j k^{B}} \delta_{k}^{B}{\overline{\left[\mathbf{A}_{r}\right.}}_{k}^{B}+c_{i j k^{M}} \delta_{k}^{M} \overline{\left[\mathbf{A}_{r}\right]_{k}^{M}},
$$

where $\delta_{i j}$ is the Kronecker symbol. The size of the system Eq. (38) is $3 \times(P+1) \times$ the number of degrees of freedom of the structure. It should be noted that the variance $\left\langle\Psi_{i}^{2}\right\rangle$ and the coefficients $c_{i j k} \bullet\left\langle\xi_{k}^{\bullet} \Psi_{i} \Psi_{j}\right\rangle$ need to be calculated only once.

Matrices $\left[\mathbf{A}_{r}\right]_{k}^{K},\left[\mathbf{A}_{r}\right]_{k}^{B}$ and $\left[\mathbf{A}_{r}\right]_{k}^{M}$ designate the block matrices $k$ of the substructures leading to the assembly matrices $\left[\mathbf{A}_{r}\right]^{K},\left[\mathbf{A}_{r}\right]^{B}$ and $\left[\mathbf{A}_{r}\right]^{M}$, and are expressed as

$$
\begin{aligned}
& {\left[\mathbf{A}_{r}\right]^{K}=\left[\begin{array}{cc}
-\frac{1}{2} T i \omega\left[\mathbf{K}_{r}\right] & {[\mathbf{0}]} \\
{[\mathbf{0}]} & {\left[\mathbf{K}_{r}\right]}
\end{array}\right], \quad\left[\mathbf{A}_{r}\right]^{M}=\left[\begin{array}{cc}
\frac{1}{2} T i \omega^{3}\left[\mathbf{M}_{r}\right] & {[\mathbf{0}]} \\
{[\mathbf{0}]} & -\omega^{2}\left[\mathbf{M}_{r}\right]
\end{array}\right]} \\
& {\left[\mathbf{A}_{r}\right]^{B}=\left[\begin{array}{cc}
\frac{1}{2} T \omega^{2}\left[\mathbf{B}_{r}\right] & {[\mathbf{0}]} \\
-i \omega\left[\mathbf{B}_{r}\right] & i \omega\left[\mathbf{B}_{r}\right]
\end{array}\right]}
\end{aligned}
$$

\section{Application}

The structure which is to be updated is a simplified version of the 'GARTEUR SM AG 19' [20]. The geometry of the structure is shown on Fig. 2. Tables 1, 2 and 3 present the geometric characteristics, the material properties and the locations of the sensors respectively.

The frequency range studied included the first fourteen modes of the structure: [5,55] Hz. A deterministic excitation was applied on the right drum and the response was measured with 24 sensors (see Fig. 3). The structure was divided into seven different groups. Groups 1 to 6 correspond to the structure and group 7 corresponds to the elastic supports. The original size of the finite element model was 1,530 DOFs. We used the reduced basis $T$ constructed in Subsection 3.2 to bring the total number of DOFs down to 53.

The only random variable was the damping of the wing. The measured damping matrix $[\mathbf{B}]^{\text {mes }}$ is:

$$
[\mathbf{B}]^{m e s}=[\overline{\mathbf{B}}]_{0}+\sum_{i=1}^{N_{g}} \alpha_{i}^{m e s}[\overline{\mathbf{B}}]_{0 i}+\delta_{6}^{m e s} \xi[\overline{\mathbf{B}}]_{06},
$$

where $[\overline{\mathbf{B}}]_{0}$ and $[\overline{\mathbf{B}}]_{0 i}$ are the deterministic damping matrix of the initial guess before the updating process, for the whole finite element model and for the each substructure $i$ respectively. In addition, $\alpha_{i}^{\text {mes }}, i=1$ to $N_{g}$ are 
Table 1

Geometric characteristics of the plate and bar models

\begin{tabular}{cccccccc}
\hline Group & 1 & 2 & 3 & 4 & 5 & 6 & 7 \\
\hline Thickness $(\mathrm{m})$ & 0.05 & 0.01 & 0.01 & 0.01 & 0.01 & 0.011 & \\
Cross section $\left(\mathrm{m}^{2}\right)$ & & & & & & & 1.E-4 \\
\hline
\end{tabular}

Table 2

The material properties used in the calculation

\begin{tabular}{ccccc}
\hline Group & Young's Modulus & Poisson's ratio & Density & Proportional damping coefficient \\
\hline$\{1, \ldots, 6\}$ & $72 \mathrm{E} 9 \mathrm{~Pa}$ & 0.3 & $2,700 \mathrm{~kg} / \mathrm{m}^{3}$ & $1 . \mathrm{E}-4$ \\
7 & $3 \mathrm{E} 2 \mathrm{~Pa}$ & 0.3 & $100 \mathrm{~kg} / \mathrm{m}^{3}$ & $1 . \mathrm{E}-4$ \\
\hline
\end{tabular}

Table 3

Position of the sensors in the $(O, \underline{x}, \underline{y}, \underline{z})$ coordinate system

\begin{tabular}{cccccccc}
\hline & \multicolumn{3}{c}{ Coordinates $(\mathrm{m})$} & & \multicolumn{3}{c}{ Measured axes } \\
\cline { 2 - 4 } \cline { 6 - 8 } Sensor & $\mathrm{X}$ & $\mathrm{Y}$ & $\mathrm{Z}$ & & $\mathrm{X}$ & $\mathrm{Y}$ & $\mathrm{Z}$ \\
\hline 1 & 0.00 & 0.00 & 0.00 & & 1 & 1 & 1 \\
2 & 0.95 & 0.00 & 0.15 & & 1 & 1 & 1 \\
3 & 1.40 & 0.00 & 0.00 & & 0 & 1 & 0 \\
4 & 1.40 & 0.00 & 0.45 & & 0 & 1 & 0 \\
5 & 1.40 & 0.20 & 0.45 & & 1 & 0 & 1 \\
6 & 1.40 & -0.20 & 0.45 & & 1 & 0 & 1 \\
7 & 0.42 & 0.93 & 0.15 & & 1 & 0 & 1 \\
8 & 0.80 & 0.93 & 0.15 & & 0 & 0 & 1 \\
9 & 0.42 & -0.93 & 0.15 & & 1 & 0 & 1 \\
10 & 0.80 & -0.93 & 0.15 & & 0 & 0 & 1 \\
11 & 0.55 & 0.62 & 0.15 & & 1 & 0 & 1 \\
12 & 0.55 & -0.62 & 0.15 & & 1 & 0 & 1 \\
13 & 0.55 & 0.27 & 0.15 & & 0 & 0 & 1 \\
14 & 0.55 & -0.279 & 0.15 & & 0 & 0 & 1 \\
\hline
\end{tabular}

nondimensional coefficients. $\delta_{6}^{\text {mes }}$ designates the coefficient of variation of the wing's damping for the measurements. The damping matrix of the model is:

$$
[\mathbf{B}]=[\overline{\mathbf{B}}]+\delta_{6}^{\bmod } \xi[\overline{\mathbf{B}}]_{6},
$$

where $\delta_{6}^{m o d}$ is the coefficient of variation of the wing's damping for the model. It should be noted that $[\overline{\mathbf{B}}]$ was constructed such that $[\overline{\mathbf{B}}]=\sum_{i=1}^{N_{g}} \alpha_{i}^{\text {mod }}[\overline{\mathbf{B}}]_{0 i}$, where $\alpha_{i}^{\text {mod }}$ are nondimensional coefficients which must be updated. The experiments were simulated using the Monte Carlo method with a statistical sample size $n=10$.

\subsection{Initial model and experimental responses}

Figure 5(a) shows graphs of the displacements for the simulated experimental data, and of the displacements calculated from the model using the one-dimensional polynomial chaos expansion of order $p=3$. These graphs confirm that the initial model did not agree with the measurements.

\subsection{Mass and stiffness updating}

The Drucker error was used in order to carry out the updating of the average mass and stiffness. The localization step identified the highest error on the wing of the structure (Group 6). The global Drucker error in that case was about $2 \%$. After a first correction step, based on a gradient method with adaptive step size, the global error dropped to $0.4 \%$ (see Table 4 ). 


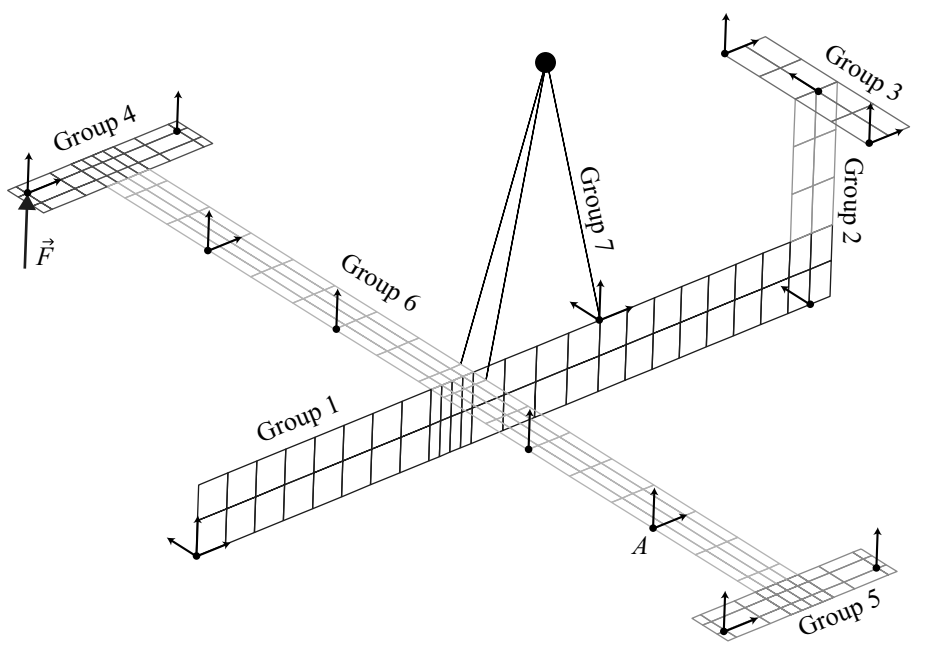

Fig. 3. The finite element plate model showing the positions of the accelerometers and excitations on the test structure.

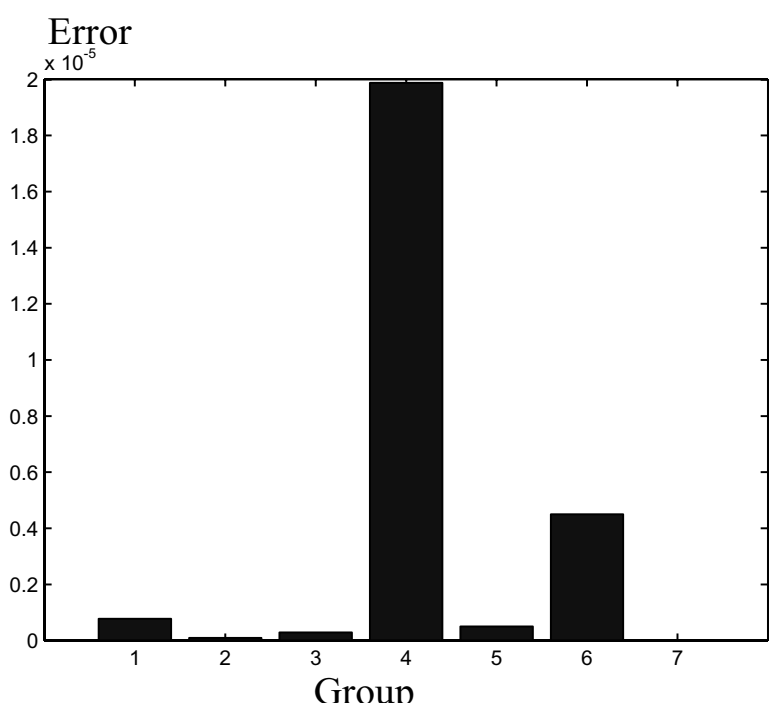

(a) Drucker error

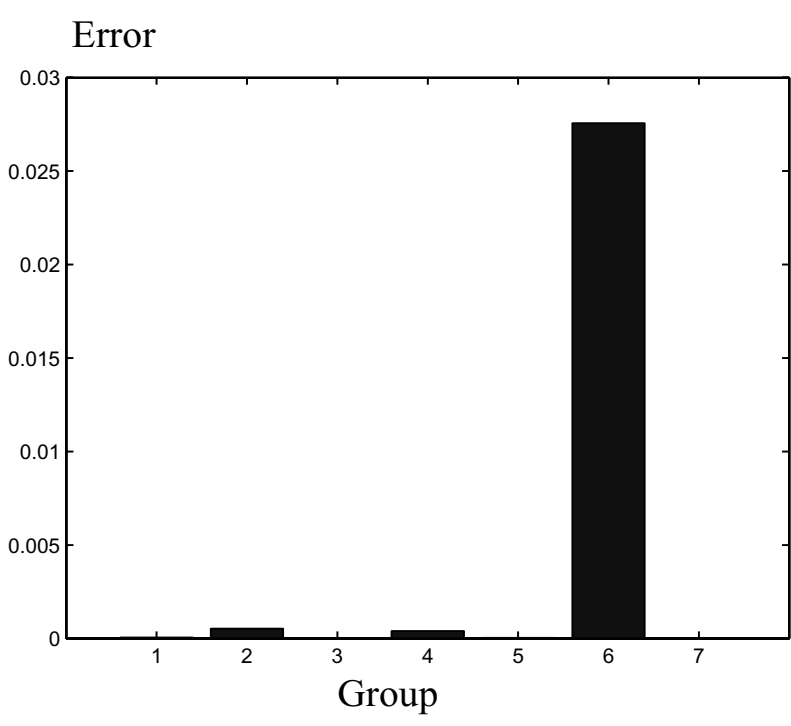

(b) Dissipation error

Fig. 4. Comparison of the localizations calculated from the Drucker error and from the dissipation error after stiffness and mass updating.

\subsection{Updating of the damping}

Figure 4 shows a comparison of the detections of local damping errors during the localization step achieved with the Drucker error (Fig. 4(a)) and with the dissipation error (Fig. 4(b)). This clearly proves the superiority of the stochastic dissipation error, which locates Group 6 (which theoretically has the most errors) correctly, while the Drucker error points toward an incorrect location (Group 4). Therefore, we carried out the updating of the wing's damping using the dissipation error, which brought the global dissipation error down to $1.4 \%$ (see Table 4). Figure 5(b) confirms that the updated model agreed with the measurements. 
Table 4

Simulated measurements parameters, initial guess prior to updating, and updated model parameters

\begin{tabular}{cccc}
\hline Measurements & Initial guess & First update (Drucker) & Second update (Dissipation) \\
\hline$\alpha_{k, 6}^{\text {mes }}=1.2$ & $\alpha_{k, 6}^{\text {mod }}=1.0$ & $\alpha_{k, 6}^{\text {mod }}=1.2$ & $\alpha_{k, 6}^{\text {mod }}=1.2$ \\
$\alpha_{m, 6}^{\text {mes }}=0.9$ & $\alpha_{m, 6}^{\text {mod }}=1.0$ & $\alpha_{m, 6}^{\text {mod }}=0.89$ & $\alpha_{m, 6}^{\text {mod }}=0.89$ \\
$\alpha_{d, 6}^{\text {mes }}=5$ & $\alpha_{d, 6}^{\text {mod }}=1.0$ & $\alpha_{d, 6}^{\text {mod }}=1.0$ & $\alpha_{d, 6}^{\text {mod }}=5.1$ \\
$\delta_{d, 6}^{\text {mes }}=0.1$ & $\delta_{d, 6}^{\text {mod }}=0.2$ & Not updated & Not updated \\
Drucker Error & $2.1 \%$ & $0.4 \%$ & Not applicable \\
Dissipation Error & Not applicable & $16.3 \%$ & $1.4 \%$ \\
\hline
\end{tabular}

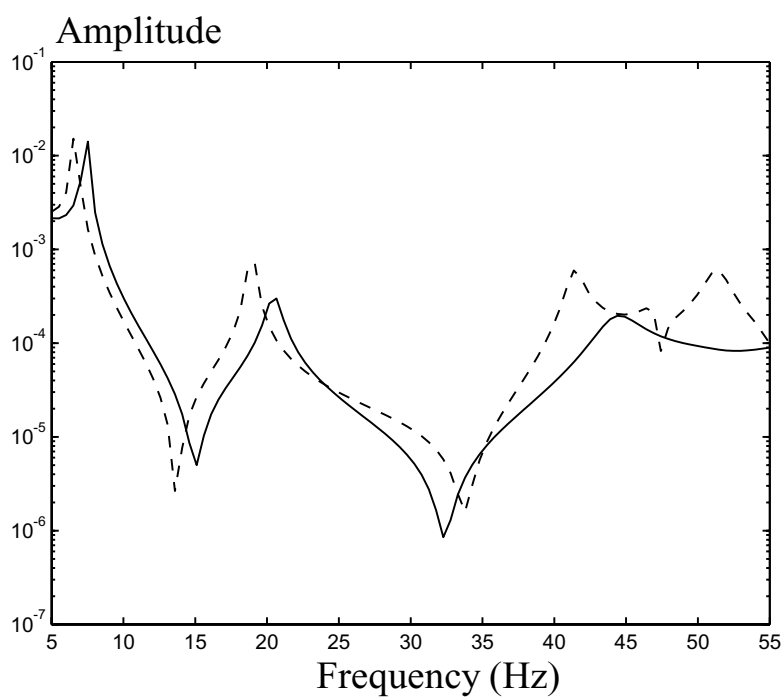

(a) Before updating

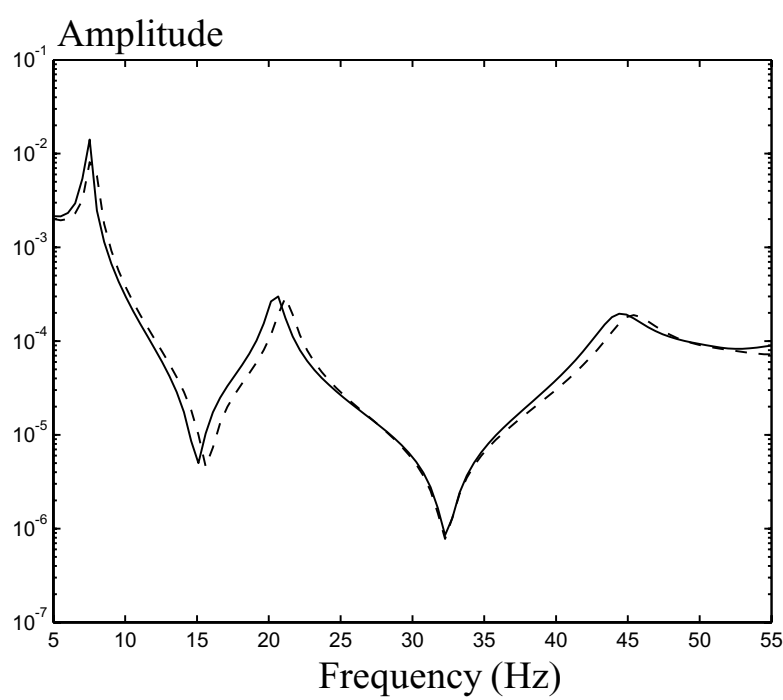

(b) After updating

Fig. 5. Displacement at Point $A$ of Fig. 3 for the experimental data (solid line) and for the model (dashed lines) as a function of the frequency.

\section{Conclusion}

In previous works, the Drucker error was successfully used to update the stiffness and mass properties of stochastic finite element models. In this paper, we studied the updating of damping in a structure using another type of error called the dissipation error. We focused on the case of uncertain measurements obtained from a family of quasi-identical structures. In order to do that, we presented an extension of the dissipation error usable in updating stochastic models.

The minimization of this stochastic error leads to a random linear system which is solved using a polynomial chaos expansion. Since the resolution of this linear system can be very costly, we proposed using a reduced basis consisting of some deterministic real eigenmodes and many static modes. This basis has the advantage of being similar to those used with the Drucker error.

This method was tested on the GARTEUR SM AG 19. We studied the case of a random damping variable used to model a viscoelastic layer on the wing. First, we updated the incorrect mass and stiffness using the Drucker error. Then, we showed that, contrary to the Drucker error, the dissipation error is effective in locating the incorrect damping. Then, the updating process was performed successfully using a gradient method with adaptive step size. Since the gradient is an explicit expression of Fields $\{U\}$ and $\{V\}$, a BFGS method could also be used without seriously affecting the computing time. 


\section{References}

[1] J. Mottershead and M. Friswell, Model updating in structural dynamics: a survey, J Sound Vib 167(2) (1993), 347-375.

[2] M. Baruch, Optimal correction of mass and stiffness matrices using measured modes, AIAA Journal 20(11) (1982), 1623-1626.

[3] A. Berman and E.J. Nagy, Improvement of a large analytical model using test data, AIAA Journal 21(8) (1983), 1168-1173.

[4] H. Berger, R. Ohayon, L. Quetin, L. Barthe, P. Ladevèze and M. Reynier, Updating methods for structural dynamics models, La Recherche Aérospatiale 5 (1991), 9-20, (in French).

[5] C. Farhat and F. Hemez, Updating finite element dynamics models using an element-by-element sensitivity methodology, AIAA Journal 31(9) (1993), 1702-1711.

[6] J. Piranda, G. Lallement and S. Cogan, Parametric correction of finite element modes by minimization of an output residual: improvement of the sensitivity method, in: Proc. IMAC IX, Firenze, Italy, 1991, 363-368.

[7] S. Lammens, M. Brughmans, J. Leuridan, W. Heylen and P. Sas, Application of a FRF based model updating technique for the validation of a finite element model of components of the automotive industry, ASME Conference, Boston, 1995, 1191-1200.

[8] P. Ladevèze and M. Reynier, FE modeling and analysis: a localization method of stiffness errors and adjustments of FE models, in: Vibrations Analysis Techniques and Application, ASME Publishers, 1989, pp. 355-361.

[9] P. Ladevèze, D. Nedjar and M. Reynier, Updating of finite element models using vibrations tests, AIAA Journal 32(7) (1994), 1485-1491.

[10] P. Ladevèze and A. Chouaki, Application of a posteriori error estimation for structural model updating, Inverse Prob 15 (1999), 49-58.

[11] P. Ladevèze, Validation and verification of stochastic models in uncertain environment through the constitutive relation error method, Internal Report 258, in French, LMT-Cachan, June 2003.

[12] P. Ladevèze, G. Puel, A. Deraemaeker and T. Romeuf, Validation of structural dynamics models containing uncertainties, Comput Methods Appl Mech Engrg 195 (2006), 373-393.

[13] A. Deraemaeker, P. Ladevèze and $\mathrm{Ph}$. Leconte, Reduced based for model updating in structural dynamics based on constitutive relation error, Comput Methods Appl Mech Engrg 191 (2002), 2427-2444.

[14] B. Faverjon, P. Ladevèze and F. Louf, Validation of stochastic structural dynamics models, CST 2006 - 8th International Conference on Computational Structures Technology, 2006.

[15] R. Ghanem and P. Spanos, Stochastic Finite Elements: A Spectral Approach, Springer, Berlin, 1991.

[16] P. Ladevèze and N. Moes, A new a posteriori error estimation for non-linear time-dependent finite element analysis, Comput Methods Appl Mech Engrg 157 (1997), 45-68.

[17] P. Ladevèze, Nonlinear Computational Structural Mechanics. New approaches and Non-Incremental Methods of Calculation, Springer, New York, 1998.

[18] G. Schueller, A state-of-the-art report on computational stochastic mechanics, Probabilist Engrg Mech 12(4) (1997), 197-232.

[19] A. Chouaki, Recalage de modèles dynamiques de structures avec amortissement, PhD Thesis, in French, LMT-Cachan, July 1997.

[20] E. Balmès and J. Wright, GARTEUR group on ground vibration testing. Results from the test of a single structure by 12 laboratories in Europe, 1997 ASME Design Engineering Technical Conferences, Sacramento, September 14-17, 1997. 

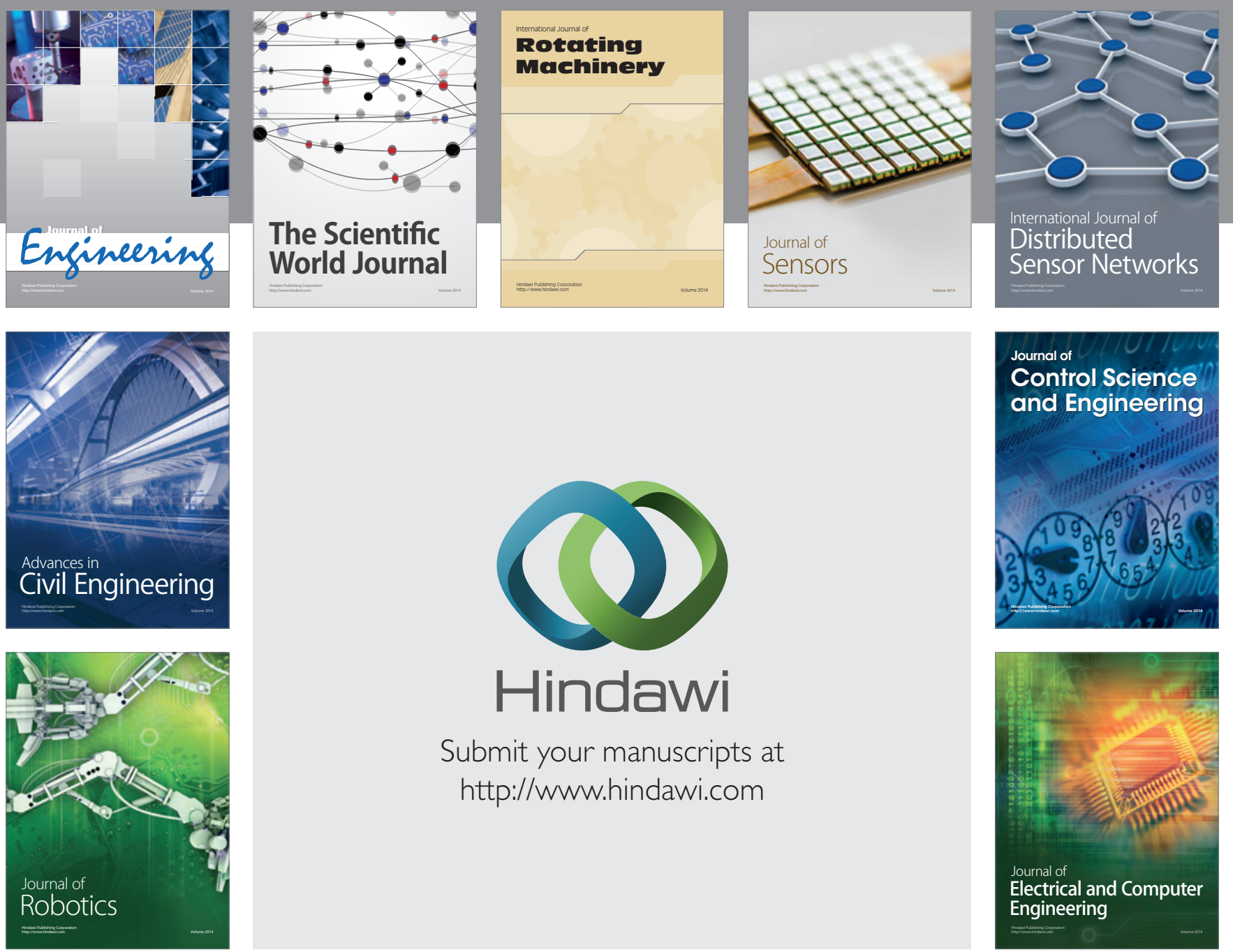

Submit your manuscripts at

http://www.hindawi.com
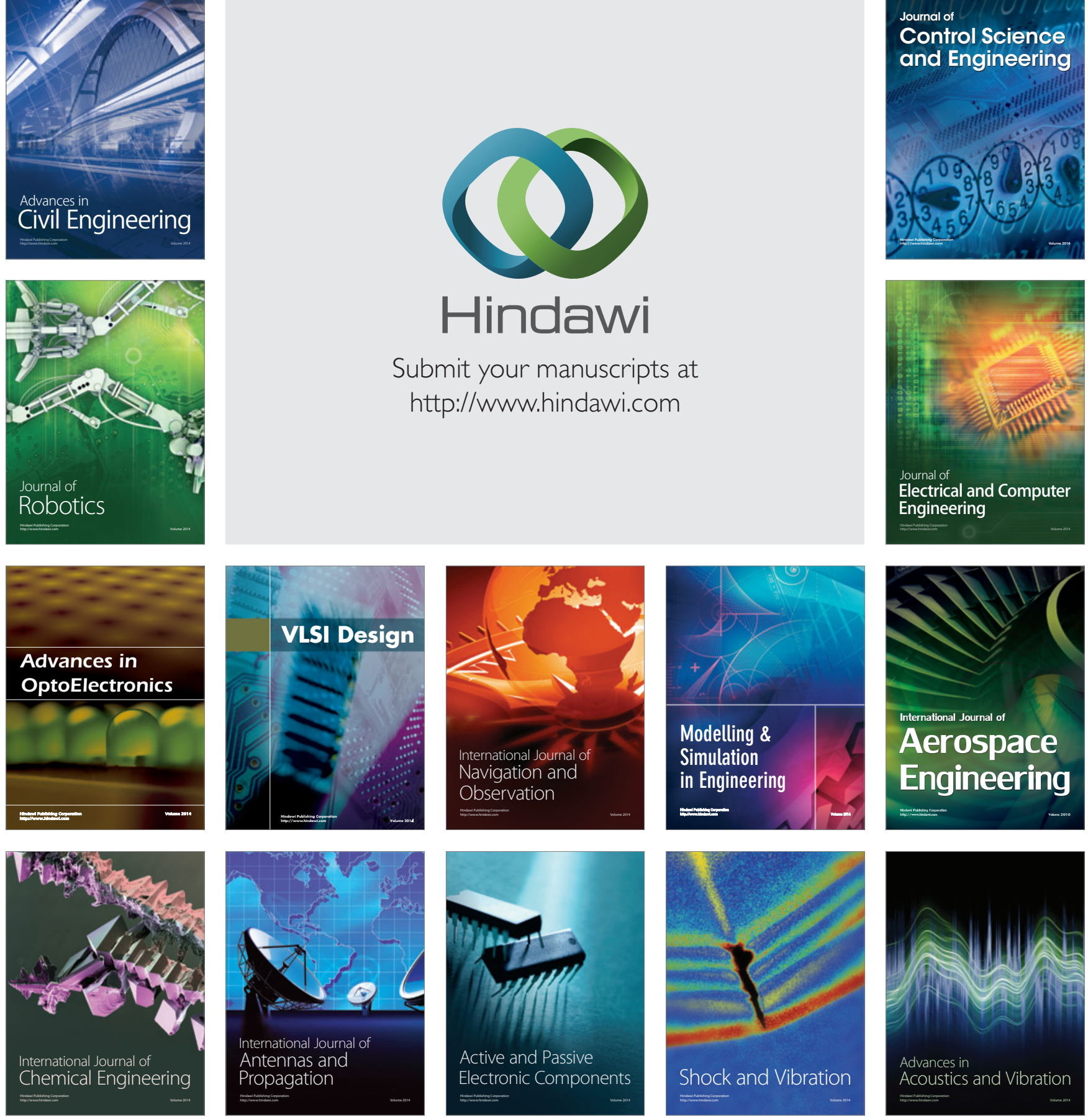